EP-107

\title{
Telemedicine in hepatology
}

\author{
Lawanya RANJAN ${ }^{1}$, Amar RANJAN*² \\ 'Engineering Management - Telecommunication, Service Stream Limited, Melbourne, Australia \\ ${ }^{2}$ Cancer Institute, All India Institute of Medical Sciences, New Delhi, India
}

Introduction: Telemedicine involves medical consultation through telecommunication facilities using online devices.

Methods: Literatures review has been done.

Results: Telemedicine includes following components. Interactive (live) Medicine: it allows patients and physicians to communicate in real-time, while the two are distant locations. They share information from one computer screen to another. Communication is encrypted and transferred. Health data like heart rate, blood pressure, glucose levels are shared. Interaction is done among patient, nearby health professionals or doctors at remote areas with a specialist doctor for diagnosis and management of certain health conditions. It may be used for second opinion or for opinion of other specialities. Many subdivisions are existing even today like 'teleradiology', telepathology. Telemedicine services are evolving for almost all branched of medicine like Mental Health, Pediatric, Dermatology, etc. Specialized Mobile Health devices: in Smartphone, the person opens the app and clicks to choose a doctor. Small scopes and other peripherals can be plugged into a mobile phone. These devices transform the phone into a pocket-sized diagnosis tool, excellent for point-of-care tests. These devices are Digital stethoscope, EKG, Pulse oximeter, Ultrasound, Blood pressure cuff, Otoscope, Dermatoscope, etc. Online Medical Centres are evolving with 24/7 online medical facilities. Setting up of telemedicine: various Video-conferencing software like OnCall Health, DrChrono HER, etc are available. Portable telemedicine kits includes a computer and mobile medical devices (ECG). Disadvantages: no clear administrative policy is available. Data leak is of concern.

Conclusions: Telemedicine is an evolving concept and is the need of hours. 